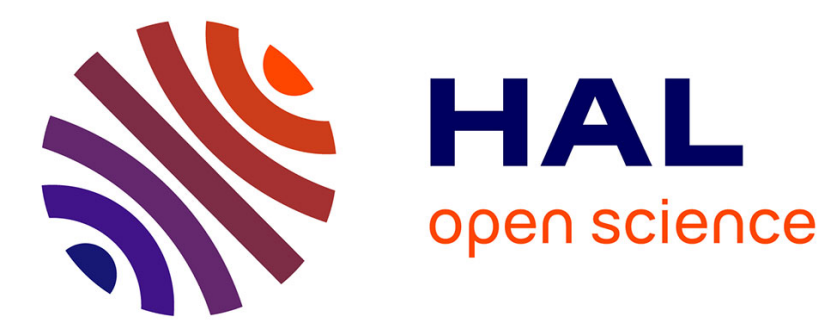

\title{
Observation of Edge Waves in a Two-Dimensional Su-Schrieffer-Heeger Acoustic Network
}

Li-Yang Zheng, Vassos Achilleos, Olivier Richoux, Georgios Theocharis, Vincent Pagneux

\section{- To cite this version:}

Li-Yang Zheng, Vassos Achilleos, Olivier Richoux, Georgios Theocharis, Vincent Pagneux. Observation of Edge Waves in a Two-Dimensional Su-Schrieffer-Heeger Acoustic Network. Physical Review Applied, 2019, 12, 10.1103/PhysRevApplied.12.034014 • hal-02371105

\section{HAL Id: hal-02371105 \\ https://hal.science/hal-02371105}

Submitted on 19 Nov 2019

HAL is a multi-disciplinary open access archive for the deposit and dissemination of scientific research documents, whether they are published or not. The documents may come from teaching and research institutions in France or abroad, or from public or private research centers.
L'archive ouverte pluridisciplinaire HAL, est destinée au dépôt et à la diffusion de documents scientifiques de niveau recherche, publiés ou non, émanant des établissements d'enseignement et de recherche français ou étrangers, des laboratoires publics ou privés. 


\title{
Observation of Edge Waves in a Two-Dimensional Su-Schrieffer-Heeger Acoustic Network
}

\author{
Li-Yang Zheng $\odot,{ }^{*}$ Vassos Achilleos, Olivier Richoux, Georgios Theocharis, and Vincent Pagneux \\ LAUM, UMR CNRS 6613, Le Mans Université, Avenue O. Messiaen, 72085 Le Mans, France
}

(Received 31 March 2019; revised manuscript received 8 July 2019; published 10 September 2019)

\begin{abstract}
In this work, we experimentally report the acoustic realization of the two-dimensional Su-SchriefferHeeger model in a simple network of air channels. We analytically study the steady-state dynamics of the system using a set of discrete equations for the acoustic pressure, leading to the two-dimensional Su-Schrieffer-Heeger Hamiltonian matrix without use of the tight-binding approximation. By building an acoustic network operating in the audible regime, we experimentally demonstrate the existence of a topological band gap. Moreover, within this band gap we observe the associated edge waves even though the system is open to free space. Our results not only experimentally demonstrate topological edge waves in a zero-Berry-curvature system but also provide a flexible platform for the study of topological properties of sound waves.
\end{abstract}

DOI: 10.1103/PhysRevApplied.12.034014

\section{INTRODUCTION}

The study of topological insulators has been attracting a lot of attention in recent years due to their appealing property for the control of wave propagation [1,2]. Among other properties, topological insulators exhibit nontrivial topological phases, leading to the existence of robust edge states at the boundaries or interfaces [3,4]. Various systems exhibiting nontrivial topological phases have been investigated [5-9]. Previous studies showed that Chern insulators [10-13] and $Z_{2}$ topological insulators [14-20] possess a nontrivial topological phase stemming from nonvanishing Berry curvatures. On the other hand, it was recently reported that a topological phase can also appear in systems even in the absence of Berry curvature $[21,22]$. This new, interesting scheme has been found in the two-dimensional (2D) Su-Schrieffer-Heeger (SSH) model, which is a 2D extension of the one-dimensional SSH chain, with alternating strengths of bonds connecting identical atoms in both the $x$ direction and the $y$ direction [23-26].

The topological phase in the 2D SSH model can be characterized by a $2 \mathrm{D}$ Zak phase, and topological edge states are consequently predicted at the boundaries of these structures. Because of the difficulty in tuning the lattice couplings at will in the quantum world, most attention has been devoted to the study of 2D SSH analogs in classical systems, including photonics [24,25], and electrical circuits [26]. For phononic systems, although several analogs of 2D SSH edge modes have been reported [27,28], the exact mapping of the 2D SSH model in a square lattice to an acoustic system has not been reported so far. The

\footnotetext{
*liyang.zheng.etu@univ-lemans.fr
}

experimental realization of the 2D SSH model in acoustics can not only provide a simple and versatile platform for the study of topological edge waves but also open perspectives for activities involving other novel topological phases, such as high-order topological insulators [29-33].

The analysis of topological insulators is usually performed starting from a discrete model with special lattice symmetry. However, most systems in different domains of physics are described by continuum models associated with partial differential equations. One of the most-popular techniques to bridge the gap between the continuous and the discrete models is the tight-binding approximation (TBA) $[34,35]$. The original idea of the TBA is to singularize discrete points in space at places where the continuous field is localized, and thus it is naturally associated with resonating scatterers. This approximation technique can be rigorously applied by use of a Wannier function basis, leading to the evaluation of delicate overlap integrals [35-37]. In practice, for the application to topological insulators where the medium is periodic, it appears that the TBA provides generic discrete equations or dispersion relations with coupling coefficients that can be fitted with results from numerical simulation of the continuous problem [38]. An alternative approach, which we use here, in the spirit of quantum graph theory [39], is to directly obtain a discrete model from a continuous system with coveted hopping coefficients by combining wave-propagation properties and geometrical characteristics of the system. One advantage of the proposed approach is that it is constructive in the sense that the hopping coefficients can be prescribed at will, as opposed to the common practice in continuous systems where the hopping coefficients are calculated $a$ posteriori (e.g., by fitting or using asymptotic 
methods) $[38,40,41]$. In addition, by avoidance of the use of resonating scatterers, the discrete model obtained can be tuned to be valid over a broad range of frequencies.

In this work, we theoretically and experimentally study an acoustic 2D SSH network composed of simply connected air channels. Using the conservation of flux at the network junctions, we derive a set of discrete equations and subsequently map the acoustic system to the 2D SSH Hamiltonian. The validity of our theoretical model is checked by comparison of the dispersion relation with numerical simulations. In addition, both our theoretical model and the numerical simulations predict the appearance of topological edge states. Then an experimental implementation of the 2D SSH is achieved in the audible regime and it contains two different boundaries, one supporting edge waves and the other not supporting them. The propagation of topological edge waves is experimentally observed, recovering the characteristic profile of the SSH edge modes and exhibiting localization only at the prescribed boundaries of the network.

\section{TWO-DIMENSIONAL SSH ACOUSTIC NETWORK}

The 2D SSH model is depicted in Fig. 1(a), where identical nodes are arranged in a square lattice with lattice constant $2 L$. The unit cell containing four nodes (marked as $\alpha, \beta, \gamma$, and $\delta$ ) is indicated by a gray-dashed box in Fig. 1(a). The intracellular (intercellular) hoppings, that is, couplings of nodes within (between) unit cells, are denoted by $s(t)$ as the red (blue) bonds in Fig. 1(a). The acoustic realization of the 2D SSH model consists of a network structure that is shown in Fig. 1(b). As can be seen, the network is composed of two types of rigid square blocks with widths $L_{1}$ and $L_{2}$ and height $H$ as shown in the inset in Fig. 1(b). By placing the two blocks centered at a square-lattice substrate with lattice constant $2 L$, we fabricate two types of air channels of width $w_{1}$ and $w_{2}$. Then, when the top of the structure is covered with an additional plate, these channels form rigidly closed waveguides for the acoustic waves and the 2D SSH acoustic network is constructed. A single unit cell of the network is marked by a gray-dashed box in Fig. 1(b), and the four junctions between the air channels correspond to the nodes of the unit cell in Fig. 1(a). The sound pressure at each junction is coupled with that at its neighboring junctions through the channels of alternating widths $w_{1}$ and $w_{2}$. Thus, this coupling between neighboring junctions can be easily tuned solely by the widths $w_{1}$ and $w_{2}$, which, as we show later, play the same role as the intercellular and intracellular hoppings in the 2D SSH model, which we call $s$ and $t$, respectively.

Our theoretical treatment is based on the following fact: as long as the widths of the air channels are much

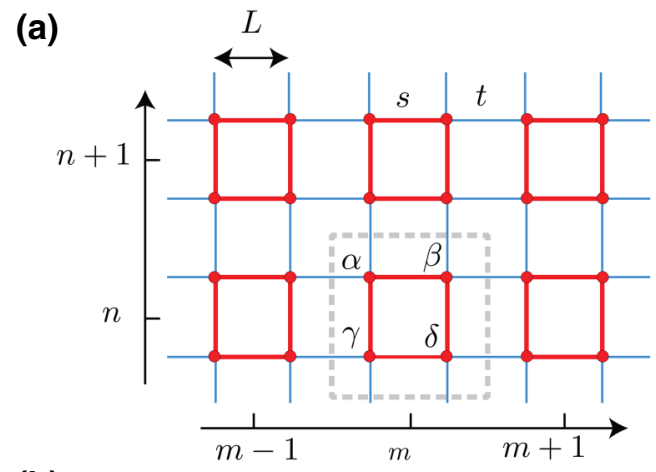

(b)

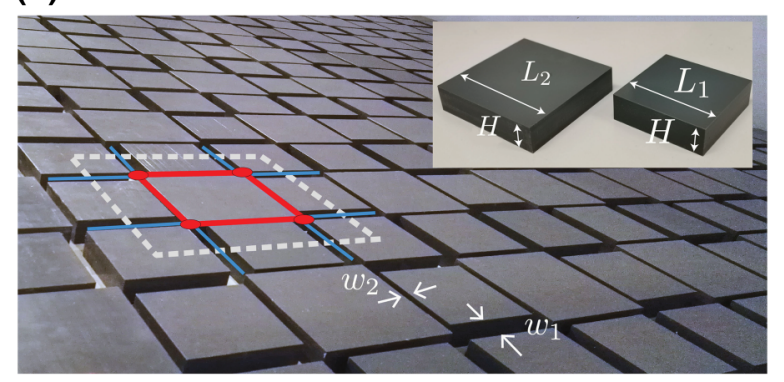

FIG. 1. (a) The 2D SSH model, which is a square lattice with lattice constant $2 L$. The unit cell containing four nodes $\alpha, \beta, \gamma$, and $\delta$ is highlighted by a gray-dashed box. The red and blue bonds represent the intracellular and intercellular couplings. (b) A view of the 2D acoustic network, which is a realization of the 2D SSH model for sound waves. The inset shows the two building blocks of the acoustic network. In the network, the intracellular and intercellular couplings can be achieved by changing the widths $w_{2}$ and $w_{1}$ of the air channels.

smaller than the channel length (i.e., $w_{1}, w_{2} \ll L$ ) soundwave propagation between junctions can be well described if we assume monomode propagation [42,43]. To derive our discrete model we label the center of each unit cell using the normalized coordinates $m$ and $n$ as shown in Fig. 1(a). Considering the unit cell at position $(m, n)$ and using the continuity of flux at each of the four junctions [44], we derive the following system of discrete equations describing the sound pressure at each junction:

$$
\begin{aligned}
& \varepsilon p_{\alpha}^{m, n}=t p_{\beta}^{m-1, n}+s p_{\beta}^{m, n}+t p_{\gamma}^{m, n+1}+s p_{\gamma}^{m, n}, \\
& \varepsilon p_{\beta}^{m, n}=s p_{\alpha}^{m, n}+t p_{\alpha}^{m+1, n}+t p_{\delta}^{m, n+1}+s p_{\delta}^{m, n}, \\
& \varepsilon p_{\gamma}^{m, n}=t p_{\delta}^{m-1, n}+s p_{\delta}^{m, n}+s p_{\alpha}^{m, n}+t p_{\alpha}^{m, n-1}, \\
& \varepsilon p_{\delta}^{m, n}=s p_{\beta}^{m, n}+t p_{\beta}^{m+1, n}+s p_{\gamma}^{m, n}+t p_{\gamma}^{m, n-1} .
\end{aligned}
$$

In Eqs. (1a)-(1d), $p_{i}$, with $i=\alpha, \beta, \gamma, \delta$, is the pressure at each junction, $t=w_{1} /\left(w_{1}+w_{2}\right)$ is the intercellular hopping coefficient, $s=1-t$ is the intracellular coefficient, and the "energy" term $\varepsilon=2 \cos 2 \pi f L / c$ (where $c$ is the speed of sound in air) depends on both the length of each channel $L$ and the frequency $f$. To obtain the corresponding dispersion relation, we seek solutions in form of Bloch 
waves as $p_{i}^{m, n}=p_{i} e^{i k_{x} m+i k_{y} n}$, where $k_{x}$ and $k_{y}$ are the wave vectors along the $x$ and $y$ directions in the first Brillouin zone, as shown in the inset in Fig. 2(a). By substituting the wave solution into Eqs. (1a)-(1d), we derive the following eigenvalue equation:

$$
\left[\begin{array}{cccc}
0 & s+t e^{i k_{x}} & s+t e^{-i k_{y}} & 0 \\
s+t e^{-i k_{x}} & 0 & 0 & s+t e^{-i k_{y}} \\
s+t e^{i k_{y}} & 0 & 0 & s+t e^{i k_{x}} \\
0 & s+t e^{i k_{y}} & s+t e^{-i k_{x}} & 0
\end{array}\right] \Psi=\varepsilon \Psi
$$

where $\Psi=\left[p_{\alpha} ; p_{\beta} ; p_{\gamma} ; p_{\delta}\right]$ is the basis consisting of the pressures at the junctions marked in Fig. 1(d). It can be seen that Eq. (2) has exactly the same form as the 2D SSH Hamiltonian introduced in Refs. [23,24], which confirms that the proposed network is an acoustic realization of the 2D SSH model. Thus, here we directly bridge the interesting topological properties of the model to the acoustic realm.

At this point we emphasize the fact that the hopping coefficients in Eqs. (1a)-(1d) are directly given by the width of the channels, in great contrast to the TBA, where these coefficients are derived as overlapping integrals of wave functions. Moreover, another remarkable advantage of our proposed method is that a large variety of discrete systems with desired coupling coefficients can be exactly mapped to an acoustic network.

We obtain the dispersion relation by solving the eigenvalue problem in Eq. (2). According to our modeling there is only one free hopping coefficient $t$ since $s=1-t$. Using $t=0.714$ and $L=0.125 \mathrm{~m}$, which correspond to the experimental setup, in Fig. 2(a) we show the dispersion curves obtained by solving the eigenvalue problem in Eq. (2). The band structure is characterized by four propagating branches (gray curves) and two full gaps around 550 and $900 \mathrm{~Hz}$. To verify the theoretical results, we perform numerical calculations using a finite-element method for a network with $t=0.714, L=0.125 \mathrm{~m}$, and $w_{1} / L=0.02$ (not the same as in the experiments); the results are shown in Fig. 2(b). In this case, by comparing Figs. 2(a) and 2(b), we observe that the dispersion relations are almost identical, confirming that the wave propagation is very well described by the discrete network model. Furthermore, we also simulate a network that has the same channel width as our experimental setup (i.e., $w_{1} / L=0.2$ ); the corresponding dispersion curve is shown in Fig. 2(c). In this case, since our main assumption that $w_{1}, w_{2} \ll L$ is not well satisfied, the theoretical model and numerical calculation exhibit a shift between the two dispersion curves. However, in the low-frequency regime and around the first band gap, which is the focus of our study, the discrete network model in Eq. (2) describes the physical system quite accurately.

One essential property of the 2D SSH model is the appearance of topological edge states when the system acquires a nontrivial topological phase, which is achieved by tuning the hopping $t$. In the acoustic network, this transition occurs in the critical case when $w_{1}=w_{2}(t=0.5)$. When $w_{1}>w_{2}(t>0.5)$, the network is in the topological nontrivial phase, and can exhibit topological edge waves. Thus, our experimental setup with $t=0.714$ is designed to fall in the nontrivial phase. To predict the presence of edge modes theoretically, we calculate the dispersion relation of a supercell containing eight unit cells with open ends (assuming a zero-pressure field) [44]. The

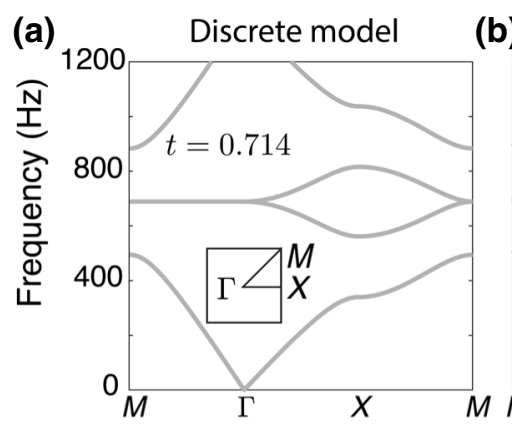

(d)

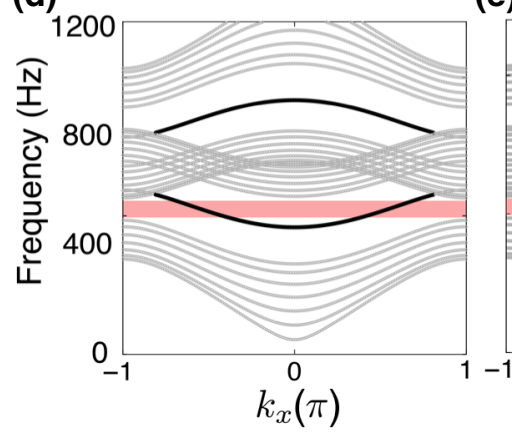

(e)
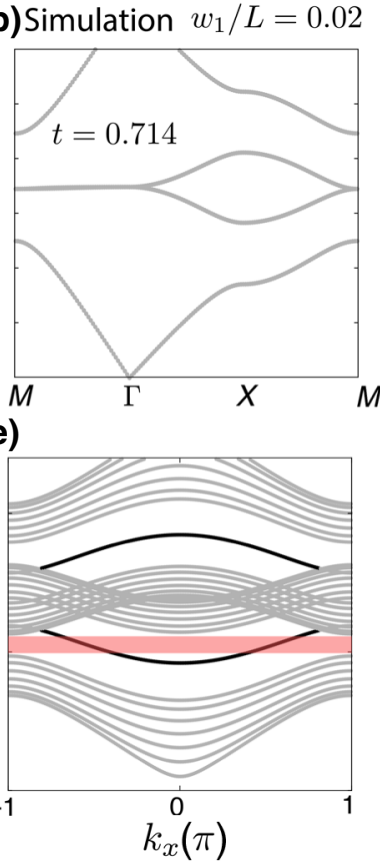

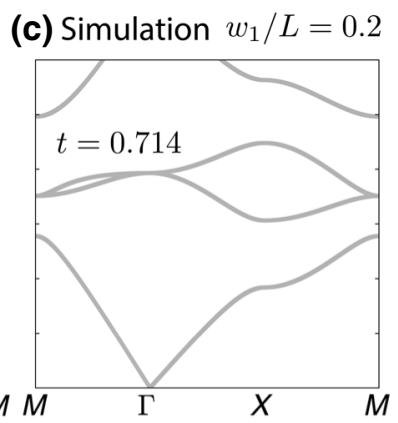

(f)

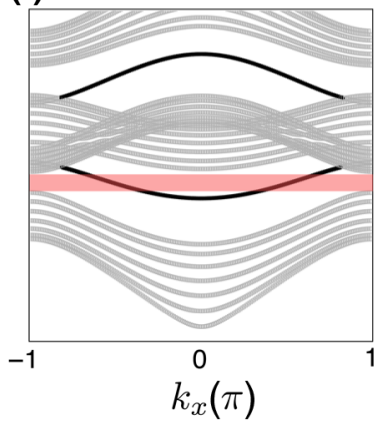

FIG. 2. The dispersion relations of the SSH network when the intercellular coupling $t=0.714$. (a)-(c) The results for bulk modes and (d) -(f) the results for edge modes by considering a supercell consisting of eight unit cells. Lines in gray represent the bulk modes and lines in black correspond to the edge modes. (a),(d) Theoretical results obtained from the network model. The inset in (a) shows the first Brillouin zone. (b),(e) Numerical results for the case of the size of air channels $w_{1} / L=0.02$. (c),(f) Numerical results for the case of the size of air channels $w_{1} / L=0.2$. 
resulting dispersion curves are depicted in Fig. 2(d). It can be seen that inside each band gap there is a degenerate edge-wave branch marked with a black line [44]. The dispersion curves for the supercell obtained by numerical simulations with zero pressure at the boundaries are shown in Figs. 2(e) and 2(f), corresponding to the same channel widths as in Figs. 2(b) and 2(c), respectively. From now on we use zero-pressure boundary conditions in all simulations. Both simulations confirm the appearance of an edge-wave branch inside the band gaps, verifying our theoretical prediction.

\section{EXPERIMENTAL IMPLEMENTATION}

We now turn to the experimental realization of the SSH network: its total size is $4 \times 2 \mathrm{~m}^{2}$ and it is constructed with building blocks of size $L_{1}=0.1 \mathrm{~m}$ and $L_{2}=0.13 \mathrm{~m}$, which leads to air channels of width $w_{1}=0.025 \mathrm{~m}$ and $w_{2}=$ $0.01 \mathrm{~m}$ as shown in Fig. 3(a). To emphasize the importance of the edge configuration, two types of edges are simultaneously investigated: one supporting edge waves [in green in Fig. 3(a)] and the other with alternative blocks that does not support edge waves [in red in Fig. 3(a)]. We focus on the first band gap around approximately $550 \mathrm{~Hz}$ as marked by the orange area in Figs. 2(d)-2(f). We experimentally identify this band gap; the results are shown in Fig. 3(b), where the measured pressure amplitude at each junction of line $x=24$ [Fig. 3(a)] as a function of frequency is plotted. For this experiment, the source is placed at position A in Fig. 3(a). To account for dissipation in the network, the viscothermal losses can be estimated by considering the length of the viscous boundary layer at around $500 \mathrm{~Hz}$. This leads to a damping term on the wave number, which is incorporated into our finite-element simulations. Experimental results are compared with numerical results in Fig. 3(c), and both confirm the existence of a band gap in the frequency range from approximately $540 \mathrm{~Hz}$ to approximately $620 \mathrm{~Hz}$. A footprint of the edge waves also appears as bright spots inside the band gap in Figs. 3(b) and 3(c) located at $y=0$ (green edge).

To better characterize the edge waves of the acoustic 2D SSH network, we perform additional experiments using a source close to the green edge [position B in Fig. 3(a)]. The normalized edge-wave profiles measured at three different lines $[x=8$ (pink), $x=18$ (orange), and $x=24$ (blue) as indicated in Fig. 3(a)] of the network are presented in Fig. 4(a). The edge wave, which is excited with a source at $570 \mathrm{~Hz}$, is revealed as the acoustic field is localized on the green edge $(y=0)$ and is decaying into the bulk. All the experimental profiles are found to exhibit a similar pattern along the $y$ axis, where the pressure field is finite in one of the sublattices and vanishes in the other one. This is the typical edge profile provided by SSH models with sublattice symmetry [45]. In our airborne experiment it is impossible to achieve an exact zero-pressure boundary condition due to a slight leakage into free space. However, as shown in Fig. 4(b), exact zero-pressure boundary conditions used in numerical simulations lead to the same profiles, confirming the robustness of the system with respect to boundary conditions. A characteristic field distribution measured at all the junctions located at $x \geq 24$ [Fig. 3(a)] is shown in Fig. 4(c) for a frequency of $582 \mathrm{~Hz}$. The corresponding numerical results are shown in Fig. 4(d), visualizing the field distribution within the whole network, in good agreement with the experiment. We clearly observe that the acoustic field is localized only at the green edges [bottom (a)

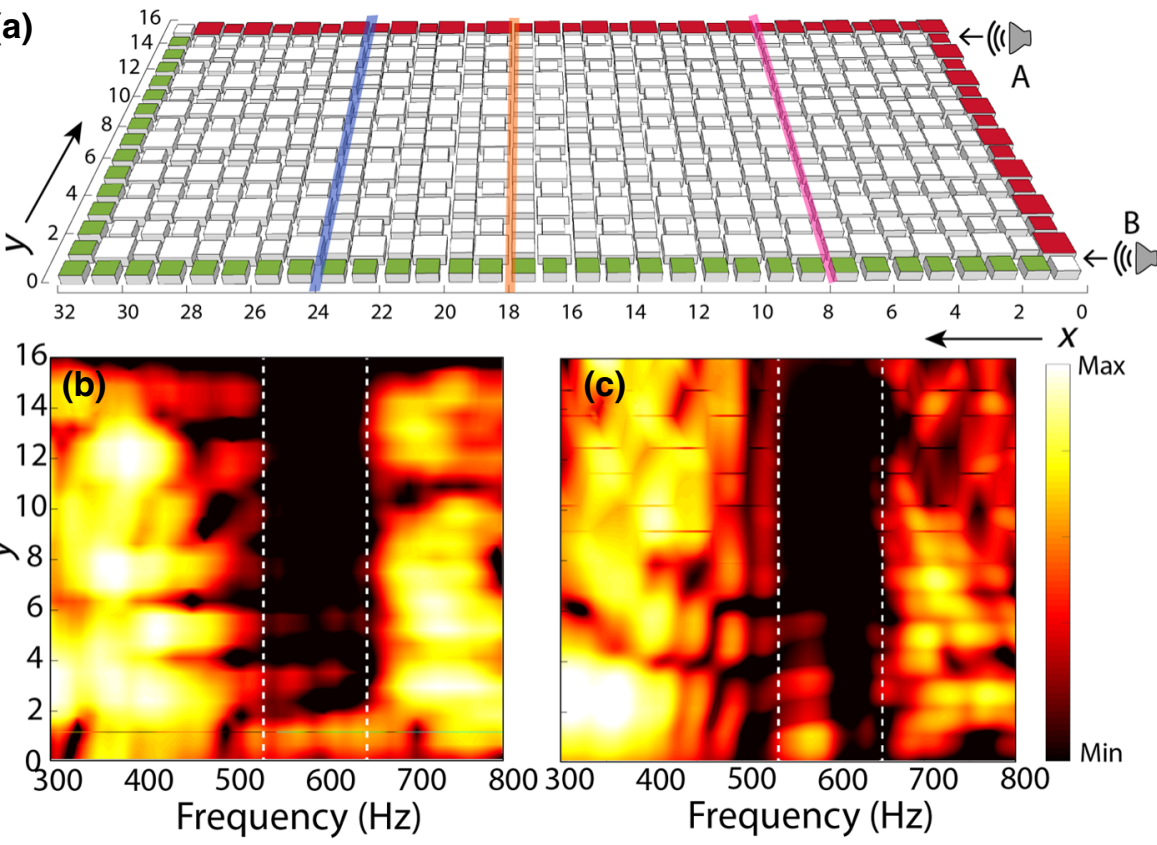

FIG. 3. Frequency-sweep experiments for the existence of a topological band gap. (a) Experimental setup. The source generated by a loudspeaker can be set at position $\mathrm{A}$ or $\mathrm{B}$ on the right side of the sample. The free edges in green (red) support (do not support) the propagation of edge waves. When the source is placed at position $\mathrm{A}$, the band gap can be measured by recording the pressures of line $x=24$ [blue line in (a)]. (b) Measured results. (c) Numerical results obtained by simulation of the experimental process. 


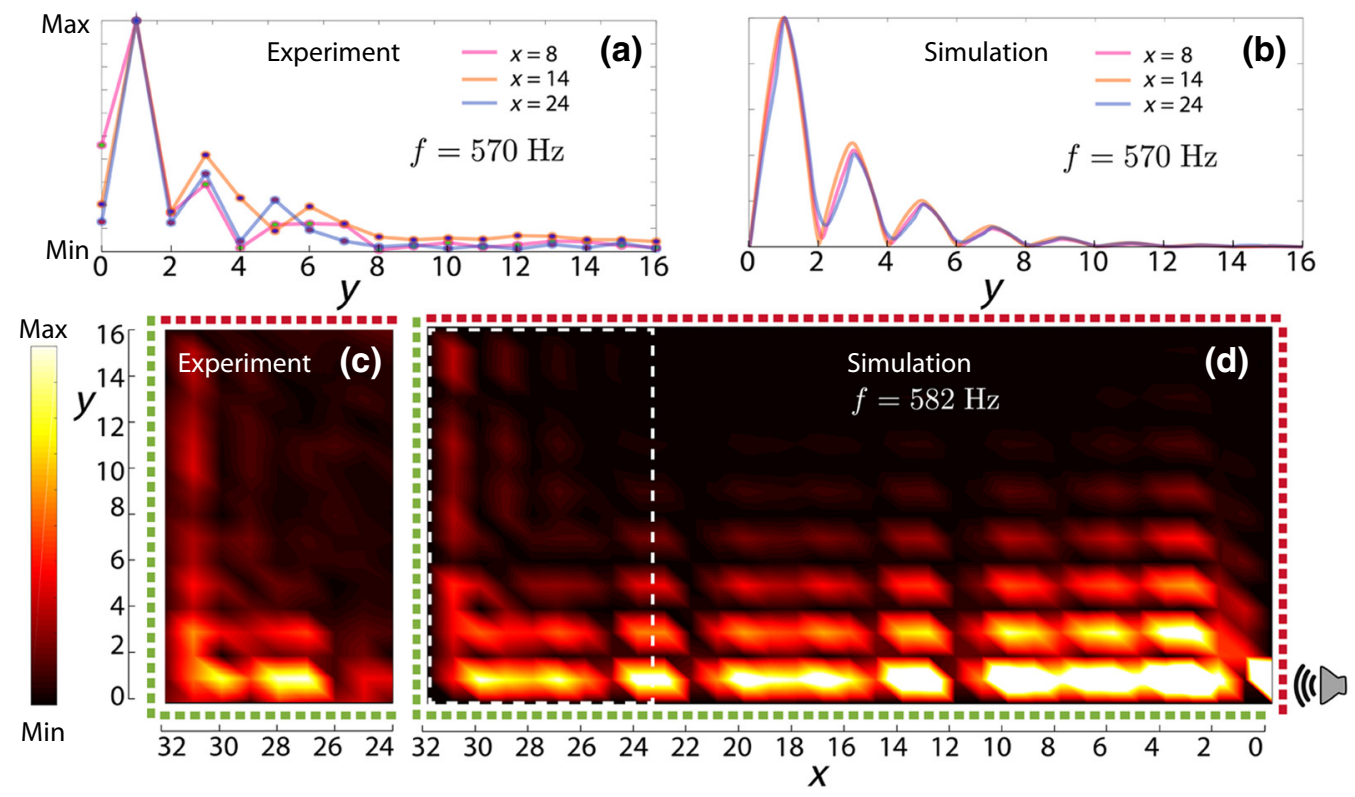

FIG. 4. Observation of edge waves. (a) The edgewave profiles of line 8 (pink), line 14 (orange), and line 24 (blue) when the frequency is $570 \mathrm{~Hz}$. (b) The edge-wave profiles obtained by numerical simulation. (c) Pressurefield distribution obtained by scanning the pressures from line 24 to line 32 in the SSH network when the frequency of $582 \mathrm{~Hz}$ is sent to the source. (d) Pressurefield distribution obtained by numerical simulation. The white-dashed box corresponds to the same area as in (c).

and left in Figs. 4(c) and 4(d)], while it vanishes at the other two edges. This is a direct consequence of the particular design of the device, which combines two different types of edges: the red edges see the bulk as trivial and the green edges see it as topological.

\section{CONCLUSION}

In conclusion, by applying a one-dimensional approximation in each connection of an acoustic network, we exactly map a continuous system to the recently proposed 2D SSH model. The latter although with zero curvature is known to support topological edge waves, which we observe in this work using an airborne experimental setup. These results show that although the system is open to free space, the edges are able to support localized waves. This work provides an acoustic demonstration of topological edge waves in a very simple system (i.e., the $2 \mathrm{D}$ SSH model) and it might be applied for experimental study of other topological phases in acoustic systems, such as higher-order topological modes.

\section{ACKNOWLEDGMENTS}

This work was funded by the Sine City project, the Acoustic Hub project, and the APAMAS project supported by Le Mans Acoustique in the framework of the Pays de la Loire region and the European Regional Development Fund.

[1] M. Z. Hasan and C. L. Kane, Colloquium: Topological insulators, Rev. Mod. Phys. 82, 3045 (2010).

[2] X.-L. Qi and S.-C. Zhang, Topological insulators and superconductors, Rev. Mod. Phys. 83, 1057 (2011).
[3] L. Lu, J. D. Joannopoulos, and M. Soljacic, Topological photonics, Nat. Photon. 8, 821 (2014).

[4] G. Ma, M. Xiao, and C. T. Chan, Topological phases in acoustic and mechanical systems, Nat. Rev. Phys. 1, 281 (2019).

[5] M. C. Rechtsman, J. M. Zeuner, Y. Plotnik, Y. Lumer, D. Podolsky, F. Dreisow, S. Nolte, M. Segev, and A. Szameit, Photonic Floquet topological insulators, Nature 496, 196 (2013).

[6] S. Yves, R. Fleury, T. Berthelot, M. Fink, F. Lemoult, and G Lerosey, Crystalline metamaterials for topological properties at subwavelength scales, Nat. Commun. 8, 16023 (2017).

[7] J. Lu, C. Qiu, M. Ke, and Z. Liu, Valley Vortex States in Sonic Crystals, Phys. Rev. Lett. 116, 093901 (2016).

[8] J.-W. Dong, X.-D. Chen, H. Zhu, Y. Wang, and X. Zhang, Valley photonic crystals for control of spin and topology, Nat. Mater. 10, 39 (2016).

[9] C. L. Kane and E. J. Mele, Quantum Spin Hall Effect in Graphene, Phys. Rev. Lett. 95, 226801 (2005).

[10] Z. Wang, Y. Chong, J. D. Joannopoulos, and M. Soljacic, Observation of unidirectional backscattering-immune topological electromagnetic states, Nature 461, 772 (2009).

[11] Z. Yang, F. Gao, X. Shi, X. Lin, Z. Gao, Y. Chong, and B. Zhang, Topological Acoustics, Phys. Rev. Lett. 114, 114301 (2015).

[12] P. Wang, L. Lu, and K. Bertoldi, Topological Phononic Crystals with One-way Elastic Edge Waves, Phys. Rev. Lett. 115, 104302 (2015).

[13] L. M. Nash, D. Kleckner, A. Read, V. Vitelli, A. M. Turnerc, and W. T. M. Irvine, Topological mechanics of gyroscopic metamaterials, Proc. Natl. Acad. Sci. 112, 14495 (2015).

[14] B. A. Bernevig, T. L. Hughes, and S.-C. Zhang, Quantum spin Hall effect and topological phase transition in $\mathrm{HgTe}$ quantum wells, Science 314, 1757 (2006). 
[15] A. B. Khanikaev, S. H. Mousavi, W.-K. Tse, M. Kargarian, A. H. MacDonald, and G. Shvets, Photonic topological insulators, Nat. Mater. 12, 233 (2013).

[16] S. H. Mousavi, A. B. Khanikaev, and Z. Wang, Topologically protected elastic waves in phononic metamaterials, Nat. Commun. 6, 8682 (2015).

[17] L.-H. Wu and X. Hu, Scheme for Achieving a Topological Photonic Crystal by Using Dielectric Material, Phys. Rev. Lett. 114, 223901 (2015).

[18] R. Süsstrunk and S. D. Huber, Observation of phononic helical edge states in a mechanical topological insulator, Sciences 349, 47 (2015).

[19] C. He, X. Ni, H. Ge, X.-C. Sun, Y.-B. Chen, M.-H. Lu, X.-P. Liu, and Y.-F. Chen, Acoustic topological insulator and robust one-way sound transport, Nat. Phys. 12, 1124 (2016).

[20] L.-Y. Zheng, G. Theocharis, V. Tournat, and V. Gusev, Quasitopological rotational waves in mechanical granular graphene, Phys. Rev. B. 97, 060101(R) (2018).

[21] C. L. Kane and T. C. Lubensky, Topological boundary modes in isostatic lattices, Nat. Phys. 10, 39 (2014).

[22] M. Xiao, G. Ma, Z. Yang, P. Sheng, Z. Q. Zhang, and C. T. Chan, Geometric phase and band inversion in periodicacoustic systems, Nat. Phys. 11, 240 (2015).

[23] F. Liu and K. Wakabayashi, Novel Topological Phase with a Zero Berry Curvature, Phys. Rev. Lett. 118, 076803 (2017).

[24] F. Liu, H.-Y. Deng, and K. Wakabayashi, Topological photonic crystals with zero Berry curvature, Phys. Rev. B 97, 035442 (2018).

[25] B.-Y. Xie, H.-F. Wang, H.-X. Wang, X.-Y. Zhu, J.-H. Jiang, M.-H. Lu, and Y.-F. Chen, Second-order photonic topological insulator with corner states, Phys. Rev. B 98, 205147 (2018).

[26] S. Liu, W. Gao, Q. Zhang, S. Ma, L. Zhang, C. Liu, Y. J. Xiang, T. J. Cui, and S. Zhang, Topologically protected edge state in two-dimensional Su-Schrieffer-Heeger circuit, Research 2019, 8609875 (2019).

[27] H. Xue, Y. Yang, F. Gao, Y. Chong, and B. Zhang, Acoustic higher-order topological insulator on a kagome lattice, Nat. Mater. 18, 108 (2019).

[28] X. Ni, M. Weiner, Andrea Alù, and A. B. Khanikaev, Observation of higher-order topological acoustic states protected by generalized chiral symmetry, Nat. Mater. 18, 113 (2019).

[29] A. M. Marques and R. G. Dias, Topological bound states in interacting $\mathrm{Su}-\mathrm{Sch}$ rieffer-Heeger rings, J. Phys.: Condens. Matter 30, 305601 (2018).

[30] B.-Y. Xie, G.-X. Su, H.-F. Wang, H. Su, X.-P. Shen, P. Zhan, M.-H. Lu, Z.-L. Wang, and Y.-F. Chen, Visualization of Higher-order Topological Insulating Phases in
Two-dimensional Dielectric Photonic Crystals, Phys. Rev. Lett. 122, 233903 (2019).

[31] X.-D. Chen, W.-M. Deng, F.-L. Shi, F.-L. Zhao, M. Chen, and J.-W. Dong, Direct observation of corner states in second-order topological photonic crystal slabs, arXiv:1812.08326 (2018).

[32] Y. Ota, F. Liu, R. Katsumi, K. Watanabe, K. Wakabayashi, Y. Arakawa, and S. Iwamoto, Photonic crystal nanocavity based on a topological corner state, arXiv:1812.10171 (2018).

[33] S. Imhof, C. Berger, F. Bayer, J. Brehm, L. W. Molenkamp, T. Kiessling, F. Schindler, C. H. Lee, M. Greiter, T. Neupert, and R. Thomale, Topolectrical-circuit realization of topological corner modes, Nat. Phys. 14, 925 (2018).

[34] N. W. Ashcroft and N. D. Mermin, Solid State Physics (Saunders College, Philadelphia, Pa., 1976).

[35] E. N. E. E. Lidorikis, M. M. Sigalas, E. N. Economou, and C. M. Soukoulis, Tight-binding Parametrization for Photonic Band gap Materials, Phys. Rev. Lett. 81, 1405 (1998).

[36] N. Marzari, A. A. Mostofi, J. R. Yates, I. Souza, and D. Vanderbilt, Maximally localized Wannier functions: Theory and applications, Rev. Mod. Phys. 84, 1419 (2012).

[37] F. B. Pedersen, G. T. Einevoll, and P. C. Hemmer, Wannier functions for the Kronig-Penney model, Phys. Rev. B 44, 5470 (1991).

[38] X. Ni, M. A. Gorlach, A. Alu, and A. B. Khanikaev, Topological edge states in acoustic Kagome lattices, New J. Phys. 19, 055002 (2017).

[39] P. Kuchment and O. Post, On the spectra of carbon nanostructures, Commun. Math. Phys. 275, 805 (2007).

[40] A. L. Vanel, O. Schnitzer, and R. V. Craster, Asymptotic network models of subwavelength metamaterials formed by closely packed photonic and phononic crystals, Europhys. Lett. 119, 64002 (2017).

[41] J. Mei, Y. Wu, C. T. Chan, and Z.-Q. Zhang, First-principles study of Dirac and Dirac-like cones in phononic and photonic crystals, Phys. Rev. B 86, 035141 (2012).

[42] C. Depollier, J. Kergomard, and J. C. Lesueur, Propagation of low frequency acoustic waves in periodic 2D-lattices of tubes, J. Sound Vib. 142, 153 (1990).

[43] P. Wang, Y. Zheng, M. C. Fernandes, Y. Sun, K. Xu, S. Sun, S. H. Kang, V. Tournat, and K. Bertoldi, Harnessing Geometric Frustration to Form Band Gaps in Acoustic Channel Lattices, Phys. Rev. Lett. 118, 084302 (2017).

[44] See Supplemental Material at http://link.aps.org/supple mental/10.1103/PhysRevApplied.12.034014 for the derivation of the equations for the network, discussion of the boundary conditions, and details on the experimental setup.

[45] J. K. Asbóth, L. Oroszlány, and A. Pályi, A Short Course on Topological Insulators (Springer, 2016). 